\section{OP0310-HPR METABOLIC ABNORMALITIES IN MEXICAN PATIENTS WITH RHEUMATIC DISEASES}

C. M. Gamboa-Alonso ${ }^{1}$, J. D. Ángulo ${ }^{1}$, R. E. Díaz-García ${ }^{1}$, G. Figueroa-Parra ${ }^{1}$, D. Á. Galarza-Delgado ${ }^{1}$, J. C. Riegatorres ${ }^{1} .{ }^{1}$ Hospital Universitario José Eleuterio González, Monterrey, Mexico

Background: Nutritional status plays an essential role in the etiopathogenesis of rheumatic diseases either as a triggering factor or as a contributor in the progression of disease activity, comorbidities and ineffective therapeutic response. ${ }^{1}$ An increased Body Mass Index (BMI) and a low lean muscle mass (LMM) have been associated to a worse clinical prognosis in rheumatic diseases.

Objectives: To describe the nutritional status and alterations in a cohort of patients with rheumatic diseases.

Methods: 658 mexican rheumatic patients from a rheumatology public center were included. Anthropometrical measurements were assessed using bio-electrical impedance analysis (BIA) Tanita. including weight, height, BMI, Body Fat Percentage and Body Fat Mass (FM), Visceral Fat (VF), MM, Total Body Water (TBW) and Bone Mass (BM) which were classified according to validated parameters as normal and abnormal.

Results: A total of 658 patients were evaluated, 368 (55.92\%) had Rheumatoid Arthritis. Table 1. The different diagnosis and anthropometric measures for each pathology are listed in Graphic 1.

More than half of the patients (68.05\%) presented an increased BMI and $85.56 \%$ a decreased MM. Graphic 2.

Conclusion: This study showed that sarcopenic obesity, defined as low MM with an increased $\mathrm{BMI}^{1}$, is a common disorder among rheumatic patients, found in more than half of our studied population. Since nutrition is a modifiable factor, important investigation in the detection and approach of metabolic abnormalities should be done.

References:

[1] Stavropoulos-Kalinoglou, A., Metsios, G. S., Panoulas, V. F., Nevill, A. M., Jamurtas, A. Z., Koutedakis, Y., \& Kitas, G. D. (2009). Underweight and obese states both associate with worse disease activity and physical function in patients with established rheumatoid arthritis. Clinical rheumatology, 28(4), 439-444.

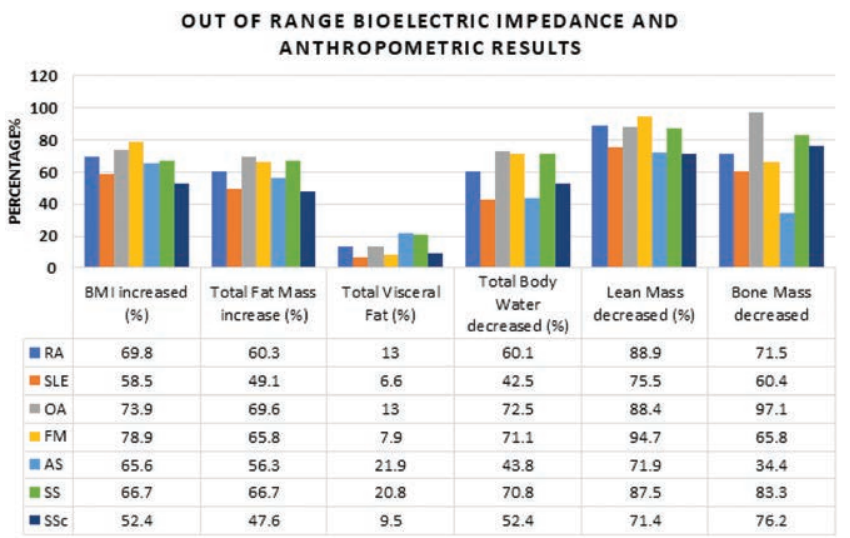

Graphic 1. RA: Rheumatoid Arthritis, LES: Systemic Lupus erythematosus, OA: Osteoarthritis FM: Fibromyalgia, EA: Spondyloarthropaties, SS: Sjögren's Syndrome, SSc: Scleroderma. A BMI $>25 \mathrm{~kg} / \mathrm{m} 2$ was classified as overweight and $>30 \mathrm{~kg} / \mathrm{m} 2$ obesity, BFM $>35 \%$ in women and $24 \%$ in men was classified as high,as well as VF $>12 \%$; a low LMM was $<68 \%$ in women and $<75 \%$ in men, $\mathrm{BM}<2.4$ was considered low.

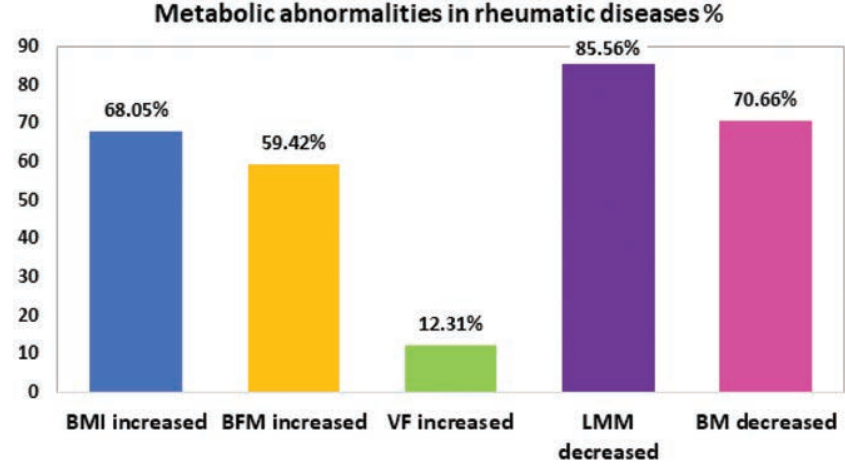

Graphic 2. Metabolic abnormalities in rheumatic diseases. BMI= Body Mass Index; BFM= Body Fat Mass; VF= Visceral Fat; LMM= Lean Muscle Mass; BM= Bone Mass

Disclosure of Interests: None declared

DOI: 10.1136/annrheumdis-2020-eular.5820

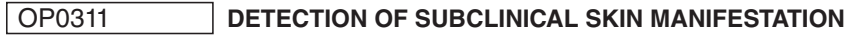 IN PATIENTS WITH PSORIASIS AND PSORIATIC ARTHRITIS BY FLUORESCENCE OPTICAL IMAGING}

A. Schmidt ${ }^{1}$, A. M. Glimm ${ }^{1}$, P. Hoff ${ }^{1}$, G. Schmittat ${ }^{1}$, G. R. Burmester ${ }^{1}$, J. Klotsche ${ }^{2,3}$, S. Ohrndorf ${ }^{1}{ }^{1}$ Charité - Universitätsmedizin Berlin, Department of Rheumatology and Clinical Immunology, Berlin, Germany;

${ }^{2}$ Deutsches Rheuma-Forschungszentrum (DRFZ) Berlin, A Leibnitz Institute, Berlin, Germany; ${ }^{3}$ Charité - Universitätsmedizin Berlin, Institute for Social Medicine, Epidemiology and Health Economics, Berlin, Germany

Background: Fluorescence optical imaging (FOI) as new imaging technique enables visualization of an impaired microcirculation in both hands caused by joint inflammation. A detection of psoriatic skin inflammation which may also signify an altered vessel composition via FOI has not yet been examined.

Objectives: The aim of the present study was to investigate potential subclinical skin inflammation in both hands of psoriasis (Pso) and psoriatic arthritis (PsA) patients in comparison to rheumatoid arthritis (RA) and healthy individuals by FOI, and to correlate these findings with cardiovascular risk factors or events, since a connection to Psoriasis skin involvement is assumed.

Methods: FOI scans of patients with Pso and PsA as well as RA and healthy subjects were analyzed retrospectively to detect subclinical skin enhancement in both hands that did not clinically show overt psoriasis skin changes. According to the 'fluorescence optical imaging activity score' (FOIAS) (1) used for evaluation of joint enhancement so far, a standardized definition was set in order to describe the degree of skin enhancement via a semi-quantitative (0-3) score (see Figure). The score was applied for the first third of the FOI exam sequence $(0-120 \mathrm{sec}$.). To be scored as potential subdermal skin enhancement, it had to be localized on the back of the hands without relationship to an underlying joint or blood vessel since the ICG enhancement was then most likely localized in the area of the (sub)dermis. Using this analysis method, we further characterized the patterns and sorted the scans into the groups PsA/Pso, RA and healthy controls to compare these with the final physician's diagnosis. Furthermore, cardiovascular risk factors (e.g. obesity, smoking status, hypertension) were collected and correlated to imaging findings.

Table 1. Bioelectric impedance and anthropometric results in Rheumatic diseases

\begin{tabular}{|c|c|c|c|c|c|c|c|}
\hline & RA & LES & OA & $\mathrm{FM}$ & EA & SS & SSc \\
\hline $\mathrm{N}$ & 368 & 106 & 69 & 38 & 32 & 24 & 21 \\
\hline Female & $335(91)$ & 98 (92.5) & 63 (91.3) & $38(100)$ & $19(59.4)$ & $23(95.8)$ & $21(100)$ \\
\hline Hight & $1.56(0.07)$ & $1.58(0.07)$ & $1.56(0.09)$ & $1.57(0.06)$ & $1.62(0.1)$ & $1.57(0.06)$ & $1.57(0.06)$ \\
\hline Age & $51.71(12.29)$ & $37.61(12.57)$ & $58.67(10.27)$ & $48(11.43)$ & $44.1(14.93)$ & $55.79(12.90)$ & $48.33(9.51)$ \\
\hline Weight (kg) & $70.02(14.94)$ & $69.1(16.77)$ & 70.34 (16.63) & $71.82(12.56)$ & $71.43(16.55)$ & $70.82(15.53)$ & $62.9(18.18)$ \\
\hline Total fat (\%) & $35.99(8.60)$ & $32.78(10.43)$ & $37.11(7.70)$ & $38.06(6.48)$ & $30.38(12.4)$ & $38.02(9.09)$ & $31.98(8.68)$ \\
\hline Total fat $(\mathrm{kg})$ & $26.06(10.81)$ & 24.41 (12.31) & $27.13(11.16)$ & $28.08(9.47)$ & $22.97(12.56)$ & $28.12(12.12)$ & $21.28(10.52$ \\
\hline Total body water (\%) & $44.71(6.06)$ & $47.41(7.41)$ & $43.45(4.31)$ & 43.21 (3.82) & 49.59 (10.47) & $42.99(5.74)$ & $46.87(4.99)$ \\
\hline Visceral fat & 8.69 (3.68) & $6.33(4.37)$ & $9.71(3.90)$ & $8.32(3.06)$ & $8.28(4.85)$ & $9.21(4.32)$ & $7.27(4.19)$ \\
\hline Lean mass (\%) & $60.75(8.18)$ & $63.71(9.98)$ & 59.67 (7.75) & $58.82(6.14)$ & 65.98 (11.77) & $58.47(9.21)$ & $66.3(11.38)$ \\
\hline Lean mass (kg) & $41.68(6.13)$ & $42.86(6.70)$ & $41.14(6.98)$ & 41.54 (3.48) & $45.92(8.74)$ & $40.11(4.74)$ & $40.13(6.92)$ \\
\hline Bone mass & $2.38(0.79)$ & $2.69(1.40)$ & $2.64(2.42)$ & $2.32(0.5)$ & $2.79(1.19)$ & $2.14(0.21)$ & $2.48(1.16)$ \\
\hline BMI & $28.69(6.15)$ & 27.79 (6.52) & $28.72(5.5)$ & $29.36(5.19)$ & $27.41(6.55)$ & 28.6 (5.98) & $25.58(7.40)$ \\
\hline
\end{tabular}

RA: Rheumatoid arthritis, SLE: Systemic Lupus Erythematosus, OA: Osteoarthritis, FM: Fibromyalgia, EA: Spondyloarthropaties, SS: Sjogren Syndrome, SSc: Scleroderma 